\title{
STRUCTURE OF THE WEAK INTERACTION
}

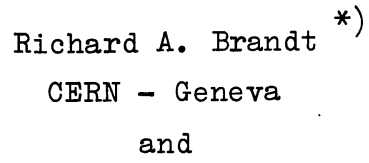

New York University, New York

and

Patrizio Vinciarelli

New York University, New York

\section{A B S T R A C T}

The interaction of electric-charge-currents ( $J$ ) and magnetic-charge-currents ( $\tilde{J}$ ) with the electromagnetic field $(\mathrm{A}, \widetilde{\mathrm{A}})$ is generalized by introducing electrically charged massive vector and axial fields $\left(W^{ \pm}, \widetilde{W}^{ \pm}\right.$) coupled to electric charge changing, magnetically neutral $\left(\mathrm{K}^{ \pm}\right)$and magnetically charged $\left(\widetilde{\mathrm{K}}^{ \pm}\right)$, $\mathrm{V}-\mathrm{A}$ currents. The resulting theory embodies (and predicts the strength of) the $C$ and $P$ violating convertional weak interaction and the still weaker $T$ violating interaction.

*) Alfred P. Sloan Foundation Fellow. 
Weak and electromagnetic interactions have many properties in common : they both apply, in a universal way, to leptons as well as hadrons ; furthermore, in a field-theoretical description, they both are vector in type. On the other land, the weak interaction is much weaker than the electromagnetic and violates symmetries which are conserved by the latter. Hence, they are usually classified as distinct, uncorrelated forces of nature.

The content of this note is highly speculative. We want to present the basic ideas of a theory in which the weak force is simply a weak manifestation of a "generalized electromagnetism". The strength of the weak interaction and its violation of $P$ and $C$ are accounted for, as well as the CP violation responsible for the $\mathrm{K}_{\mathrm{I}}^{\mathrm{O}} \rightarrow 2 \pi$ decay. Moreover, criteria of symmetry and elegance of the theory appear to us to be well satisfied.

By "electromagnetism" we do not mean "conventional electromagnetism". The latter is the theory in which the electric current is the only source of the electromagnetic field. It has been known for long 1) that symmetry considerations and the desire to understand the empirical charge quantization 2) would strongly suggest the coupling of the electromagnetic field also to a magnetic current (associated with a magnetic monopole carried by "magnetons"). The great success of conventional electromagnetism and the failure of experimental searches for magnetons 3 ) are then to be attributed to the heavy mass of these particles which makes their contribution as virtual states in quantum electrodynamics negligible and their production impossible at the present energies. These facts, however, cannot be used to argue against the existence of magnetons since the theory itself, by fixing the strength of the monopole coupling, predicts them.

Accepting the existence of a magnetic current, we shall include its contribution to the electromagnetic interaction Lagrangian density 4$), 5)$ :

$$
l_{\text {en }}=e J_{\mu} \Lambda^{\mu}+\tilde{\varepsilon} \tilde{J}_{\mu} \tilde{A}^{r}
$$

Let us explicitly observe that $J$ and $\tilde{J}$ are vector currents with the conventional space, time and charge parities of first class currents; $\widetilde{A}$ has space and time parities opposite to A, since $\tilde{J}$ is the source to the dual of the electromagnetic tensor $F_{\mu \nu}$; the electric and magnetic coupling constants (e, $\tilde{e}$ ) satisfy the Dirac quantization condition 2). The consistency of these statements follows from the quantum field theory of both electric and magnetic charges which has been developed in the last decade 2$), 4), 6)$. 
We assume the existence of dually charged particles (dyons) and that the sign of $e_{\bullet} \tilde{e}$ is the same for each particle. Then electromagnetism does not possess parity or time reversal invariance.

Here, we would like to introduce a simple and natural extension of (1) 5) :

$$
\begin{aligned}
l=e\{J \cdot A & \left.+\left[K^{+}+K_{s}^{+}\right] \cdot W^{-}+\text {h.c. }\right\}+ \\
& +\tilde{e}\left\{\tilde{J} \cdot \tilde{A}+\left[\tilde{K}^{+}+\tilde{K}_{5}^{+}\right] \cdot \tilde{W}^{-}+\text {h.c. }\right\}
\end{aligned}
$$

The generalization is motivated by the speculation that conservation of charges at electric and magnetic current-field vertices might also be realized by having the field carry a charge, in particular electric charge ${ }^{7)}$. We further speculate that charged fields have non-vanishing masses. Hence, the W field is not subject to any restriction from gauge invariance and couples to axial as well as vector currents 8 ). The new massive vector boson fields $\widetilde{W}^{ \pm}$have the same relation to $W^{ \pm}$as $\widetilde{A}$ has to A. The $\widetilde{\mathrm{K}}^{ \pm}$currents have the same electric charge properties as $\mathrm{K}^{ \pm}$and the same magnetic charge properties as $\tilde{J}$.

To illustrate the implications of (2) let us consider the contribution ( $l_{\text {partia] }}$ ) to the Lagrangian $(\ell)$ due to some spin $1 / 2$ fields (neutrino $\psi_{2}$, electron $\psi_{e}$, magneton $\psi_{m}$ and dyon $\psi_{d}$ ):

$$
\begin{aligned}
l_{\text {partial }} & =e\left\{\bar{\psi}_{l} \gamma_{2} \psi_{2} A_{2}+\bar{\psi}_{d} \gamma_{2} \psi_{d} A_{2}+\bar{\psi}_{e} \gamma_{2}\left[1+\gamma_{5}\right] \psi_{2} w_{2}^{-}\right\} \\
+ & \tilde{e}\left\{\bar{\psi}_{m} \gamma_{2} \psi_{m} \tilde{A}_{\nu}+\bar{\psi}_{d} \gamma_{2} \psi_{d} \tilde{A}_{\nu}+\bar{\psi}_{d} \gamma_{2}\left[1+\gamma_{5}\right] \psi_{e} \tilde{\omega}_{2}-\right\}+h_{1} c .
\end{aligned}
$$

Equation (3) gives rise, in particular, to electron-neutrino and electron-electron elastic scatterings. Although both processes are of order $e^{2}$ in the electric coupling constant, the amplitudes, because of the propagator, differ by several orders of magnitude. The magnetic (hyperstrong) part of (3) originates self-energy for the $W$ and A fields 9). A very rough estimate based on a comparison between electromagnetic mass differences, hadronic masses, electric, strong and magnetic (hyperstrong) coupling constants consistently yields about II $\sim 100 \mathrm{GeV}$. This should approximately be the value of the $W$ mass ${ }^{10)}$. We, therefore, obtain $e^{2} / \mathrm{M}_{\mathrm{W}}^{2} \sim 10^{-5} \mathrm{M}_{\mathrm{p}}^{-2} \sim \mathrm{G} / \sqrt{2}$, and electronneutrino scattering, as generated by (3), is a "weak" process 11). We have, in fact, roughly predicted the strength of the weak interaction. On the other hand, gauge invariance, by making the self-energy of the photon field vanish in the limit of zero momentum transfer, keeps magneton loops from significantly affecting the photon propagator 12). As a result, electron-electron scattering remains an "electromagnetic" process. 
Since the presently observed particles carry no magnetic charge, the hyper strong part of the Lagrangian can only manifest itself via virtual magnetic loops. We have already seen that these are responsible for the mass of the $W$ boson. Is the hyperstrong Lagrangian giving any other measurable effect ?

Magnetic loops build the W-A-W vertex, a T violating coupling which can be phenomenologically described by associating an electric dipole with the intermediate vector boson. We take the strength of this coupling to be of order e (the smallness of this value should be attributed to the localization in space of the magnetic loops). Hence, "generalized electromagnetism" provides us also with a small CP violation which we can hold responsible for $\mathrm{K}_{L}^{0} \rightarrow 2 \pi$ decay.

Although we know some properties of the total currents $K$ in the Lagrangian (2), we are far from being able to unequivocably state what the $\tilde{K}^{\prime} s$ are. If we were to subscribe to the idea of a complete electromagnetic theory of particles, we would say that the $\tilde{K}^{\prime} s$ are sums of different contributions of the type coming from different fundamental ( $\operatorname{spin} 1 / 2$ ) fields; we would say that " $l$ is the interaction Lagrangian of the world", that hadrons are bound states of those fundamental fields, and that hadronic interactions are nothing but a particular manifestation 13) of (2), as are weak interactions. At present, there is little evidence to support such a possibility, although we are certainly not insensitive to its aesthetical appeal.

\section{ACKNOWLEDGMENTS}

We thank Sidney Coleman for several useful discussions. 


\section{FOOTNOTES AND REFERENCES}

1) P.A.M. Dirac, Proc.Roy.Soc. A133, 60 (1931); Phys.Rev. I4, 817 (1948).

2) The existence of a single magneton with magnetic charge $\tilde{e}$ implies, via the quantization condition $\tilde{e} \tilde{e}=4 \pi \mathrm{n}$, that all electric charges in nature are multiples of a basic unit. For a more precise statement, see :

J. Schwinger, Phys.Rev. 173, 1536 (1968) and

D. Zwanziger, Phys.Rev. 176, 1489 (1968).

3) For a review of experimental searches, see :

E. Amaldi : "Old and New Problems in Elementary Particles", G. Puppi Ed., Academic Press, New York (1968).

4) We are adopting the two-potential formalism of :

N. Cabibbo and E. Ferrari, Nuovo Cimento 23, 1147 (1962). Of course, there is still only one photon.

5) The Lagrangians written in this paper are only meant to indicate couplings. They need not lead to the correct equation of motion by a variational principle. The complete Lagrangians of Ref. 4) involve path dependent matter fields. We shall discuss the complete formalism in a forthcoming paper.

6) S. Weinberg, Phys.Rev. 138, B988 (1965);

J. Schwinger, Phys. Rev. 144, 1087 (1966).

B. Zumino in "Strong and Weak Interactions - Present Problems", A. Zichichi Ed., Academic Press, New York (1966).

7) Still greater symmetry can be achieved by the addition of magnetically charged vector boson fields coupled to suitable currents. Such an addition does not affect our discussions or conclusions.

8) One might also consider including couplings to currents with different C, P, T parities.

9) Virtual magnetic loops are connected to ordinary non-magnetic processes $v i a \quad A \leftrightarrow \widetilde{A}$ and $W \leftrightarrow \widetilde{W}$ transitions.

10) Thus $[$ hadronic electromagnetic mass shift $) / e^{2} / 4 \pi \square \simeq[$ hadronic mass $\left.) / g^{2}\right] \simeq$ $\simeq\left[(w\right.$ mass $) /\left(\tilde{e}^{2} / 4 \pi \square\right.$, where $g$ is a typical strong interaction coupling constant.

11) The observed strength of the weak interaction fixes the intermediate boson mass to be $\mathrm{M}_{\mathrm{W}}^{2}=\sqrt{2} 4 \pi \alpha \mathrm{G} \approx 10^{4} \mathrm{GeV}^{2}$. This value has been previously suggested by: G. Segré, Phys.Rev. 173, 1730 (1968), in an attempt to obtain finite mass differences within isospin multiplets.

12) The scale of variation, in the momentum transfer dependence of the self-energy, is set by the heavy magneton mass.

13) Observe that $\tilde{e} \tilde{e} / 4 n$ is of the order of the strong coupling constant. The relation in footnote 10) becomes quite symmetric if $g^{2} \simeq \tilde{e} \tilde{e} / 4 \pi$ is used. 